\title{
Better training, Better care: Medical Procedures Training Initiative
}

Rachelle Shafei

NHS

\begin{abstract}
Training in procedures has been identified as the top priority for core medical trainees (GMC trainee survey 2011). Current practice relies on each trainee being lucky enough to encounter each procedure during clinical rotations and during on-calls. Where trainees are not lucky enough, they are entering their registrar years without the skills to efficiently lead the medical 'on-take'.(1) This can lead to delays in patient diagnosis or treatment. Because a single delay can easily burgeon into a lengthy series of multiple delays, this can lead to an associated prolongation of patient stay.(3)
\end{abstract}

Both confidence and competence in practical procedures can be increased with a procedure bleep system. A dedicated procedure bleep, carried on a rotational basis alerts the bleep holder when a medical procedure is planned. The bleep holder then attends to observe, assist, perform, or teach the relevant procedure.

This scheme shares the opportunities for procedure exposure amongst all trainees and ensures that a good breadth of experience has been gained independent of current placement. Formal evaluation revealed that $95 \%$ (19/20) of junior trainees felt more confident and competent as a result of participation. Furthermore, consultants felt this initiative reduced the burden on the medical registrars on-call.

By ensuring our diagnostic and therapeutic interventions are conducted efficiently, we are actively reducing length of hospital stay and improving the standard of healthcare provided.

\section{Problem}

Training in procedures has been identified as the top priority for core medical trainees (1). Medical trainee curriculums stipulate procedures in which clinical independence is essential or desirable.(2) Current practice relies on each trainee being lucky enough to encounter each procedure during each clinical rotation and during on-calls. Where trainees are not lucky enough to gain experience in all of the practical procedures required, they are entering their registrar years without the skills to efficiently lead the medical 'on-take'.(1)

This lack of experience increases the time taken for diagnostic and therapeutic interventions to be accomplished. Minor delays (of hours to days) in performing practical procedures are common.(3) Because a single delay can easily burgeon into a lengthy series of multiple delays, the primary goal should be to avoid the precipitating delay at the onset.(3)

We all know that by increasing the length of hospital stay there are unintended increases in morbidity and therefore possible effects on mortality. $(3,4)$ There are also significant financial implications, with each extra night in hospital incurring additional expense. The average cost of an excess bed day is £264.(5)

\section{Background}

Previous procedure training projects have focused on lab-based procedure training. This is an artificial environment which gives trainees the opportunity to familiarise themselves with techniques before performing procedures on patients. The benefits of skills lab training are widely accepted,(6) they lead to improvement in procedural skills compared with standard or no training at all.(7) But there is sparse research into its long-term effectiveness;(6) we do not know if skills lab training results in retention of skills over time.(7)

A recent study by Thomas $S$ et al suggests that although simulation training improved procedural skills, decay in skills was found at 3-month follow-up. This suggests that simulation training should occur in close temporal proximity to times when these skills would most likely be used clinically.(8) It is known that the hands-on clinical component results in greater retention of skills than a oneoff skills lab training session.

\section{Baseline Measurement}

In our centre, a trainee survey revealed training deficiencies in those 'essential' procedures requiring independence; external pacing, insertion of pleural intercostal drain, insertion of CVP line, and pleural tap. Trainees rated themselves on both their level of experience as reflected on their portfolios and their perceived competence (backed up with evidence from online portfolios). Their level of experience and competence was rated from 1 to 5 with 1 being no prior experience, needs basic teaching and full supervision, and 5 if fully independent and able to teach others.

Most trainees have had no experience of external pacing (18/20), and require full support with the other procedures. At month 20 of 
their 24 months of core medical training (CMT), this means that despite utilising all opportunities, our trainees are not meeting the standards they need for successful completion of CMT.

See supplementary file: ds2569.pptx - "Graph 1 and table 1 baseline 'experience' and 'competency' ratings."

\section{Design}

The proposed method for increasing both confidence and competence in practical procedures is by introducing a procedure bleep system. A dedicated procedure bleep, carried on a rotational basis by each of the core medical trainees, would alert the bleep holder when the above procedures were planned anywhere in the hospital. The bleep holder then attends either to observe, assist, perform supervised, perform unsupervised, or teach the relevant procedure depending on their experience.

The bleep holder will stay on their normal ward and complete normal duties until a procedure in the hospital was being done thus minimising disruption to existing rotas. A phantom bleep will be added onto an existing bleep, thereby ensuring no additional startup or maintenance cost.

Two trainees will be available on the rota for each day, thus ensuring that if their existing ward duties of the 'first on' are too heavy to safely leave the ward, the procedure will be conducted in a timely manner by the second bleep holder.

Hands on teaching and experience has been facilitated by:

\section{i. Procedure safety form}

In order to ensure that the procedure team is safe to carry out each procedure a form must be completed by the 'home' team in advance. This will facilitate proper investigation of the full blood count, coagulation screen and stipulate exactly which samples are required.

\section{ii. Procedure equipment lists}

An equipment list, specific for each procedure will be distributed to the junior doctors on all teams. This will serve both a teaching function, and will again ensure the timely assembly of equipment and increased efficiency of the procedure itself.

iii. Procedure booklets/online resources

\section{Strategy}

PDSA Cycle 1

The scheme was presented as an idea to the entire 'medical' team including the medical and clinical directors. Potential problems with start-up costs and patient safety had been thought through and interventions were in place to overcome each. All were in favour of such a scheme although as most medical procedures were diagnostic and therefore performed on the acute medical unit (AMU), it was felt that the scheme should be restricted to AMU patients only. Furthermore, it is the delay at this stage of the patient journey which was felt to be most important to patient outcome.

PDSA Cycle 2

The scheme started with full approval and support. Each core medical trainee had rota'ed time onto the scheme, that did not conflict with existing medical duties. After initiation, brief survey of consultants, registrars, and trainees indicated that all were happy with no further changes to the initiative necessary.

\section{Results}

At Lincoln County Hospital, this scheme has shared the opportunities for procedure exposure amongst all trainees and ensured that a good breadth of experience has been gained independent of current placement.

Formal evaluation revealed that $95 \%(19 / 20)$ of junior trainees felt it was a worthwhile initiative. Moreover, the vast majority were more confident and competent as a result of participation. Chest drains, joint aspirations, and CVP line placement in particular were areas of striking improvement, with trainees now confident to perform all three independently.

In a consultant survey, they too supported the initiative in improving training, increasing the efficiency of our patients care, and in addition, felt this initiative reduced the burden on the medical registrars on-call in the long-term.

\section{Lessons and Limitations}

The sustainability of this initiative comes from its track-record of increasing the efficiency of training and of patient care. Regular reevaluation, consultant support, and trainee feedback will help to reinforce project gains in the long-term.

Transferring this initiative to other centres would be straightforward given the low running costs and the ease of 'rota' set-up. However the exact model should be adapted to each individual institution. Close monitoring of hard outcomes together with staff opinion surveys should help to shape the details of such a scheme in other centres.

The next step in ensuring long-term skills improvement and reducing the potential for decay in skills is to combine this initiative with a lab skills session. This would combine the evidence based advantages of initial learning in skills lab training with the retention of skills in clinical practice.

\section{Conclusion}

By ensuring our diagnostic and therapeutic interventions are conducted efficiently, we are actively reducing length of hospital stay, improving the standard of healthcare provided and reducing 
hospital costs.

In short, by providing better training we are also providing better care for our patients.

\section{References}

1. GMC trainee survey 2011

2. Speciality training curriculum for core medical training, August 2009, updated August 2012, Joint Royal Colleges of Physicians Training Board

3. A Sonnenberg, B Crain. Scheduling Medical Procedures: How One Single Delay Begets Multiple Subsequent Delays. Journal of Theoretical Medicine. Volume 6 (2005), Issue 4, Pages 235-239

4. Schaffer S, Garzon L, Heroux D, Korniewicz D. Infection Prevention and Safe Practice. New York: Mosby-Year Book, 1996

5. Department of Health Reference Costs, 2011-2012

6. Lund F, Schultz JH, Maatouk I, Krautter M, Möltner A, Werner $A$, et al. Effectiveness of IV cannulation skills laboratory training and its transfer into clinical practice: a randomized, controlled trial. PLoS One. 2012;7(3):e32831

7. Lynagh M, Burton R, Sanson-Fisher R. A systematic review of medical skills laboratory training: where to from here? Med Educ. 2007 Sep;41(9):879-87

8. Thomas S, Burch W, Kuehnle S, Flood R, Scalzo A, Gerard J. Simulation training for pediatric residents on central venous catheter placement: a pilot study. Pediatr Crit Care Med. 2013 Nov;14(9):e416-23

\section{Declaration of interests}

nothing to declare

\section{Acknowledgements}

Dr R Sriraman 